\title{
Global Health Education in UK Medical Schools (GHEMS) study protocol
}

Soham Bandyopadhyay', Thomas Shortland², Shavinthi W Wadanamby ${ }^{3}$, Hannah S Thomas ${ }^{4}$, Binay Gurung ${ }^{5}$, Melika Akhbari ${ }^{6}$, Isobel Trout ${ }^{7}$, Rashida Patel $^{8}$, Karisma Sharma $^{8}$, J Edward Fitzgerald ${ }^{9}$, Adrian D Smith ${ }^{10}$

${ }^{1}$ Medical Sciences Division, University of Oxford, Oxford, UK, ${ }^{2}$ Warwick Medical School, Warwick, UK, ${ }^{3}$ University of Sheffield, Sheffield, UK, 4 University of Edinburgh College of Medicine and Veterinary Medicine, Edinburgh, UK, 5 George Davies Centre for Medicine, University of Leicester, Leicester, UK, ${ }^{6}$ GKT School of Medical Education, King's College London, London, UK, 7 University of Birmingham, Birmingham, UK, ${ }^{8}$ University of Leeds, Leeds, UK, ${ }^{9}$ King's Centre for Global Health, King's College London, London, UK, ${ }^{10}$ Nuffield Department of Population Health, University of Oxford, Oxford, UK

Keywords: global health

https://doi.org/10.29392/joghr.3.e2019052

\section{Journal of Global Health Reports}

Vol. 3, 2019

\section{Background}

Global health is the study, research, and practice of medicine focused on improving health and achieving health equity for all persons worldwide. Such focuses are core tenets of modern medical practice. The General Medical Council (GMC) have published several documents that outline specific global health learning outcomes and competencies that are essential, non-elective components of UK medical school curricula. However, there is a paucity of evidence demonstrating that medical schools have integrated these mandatory global health learning outcomes within their curricula. This multicentre study aims to delineate the breadth of global health teaching during undergraduate and graduate entry medical training across UK medical schools.

\section{Methods}

This national, multi-centre study will involve obtaining timetables containing details of all medical teaching provided at undergraduate and/or graduate entry level at UK medical schools for the academic year 2018/2019. Collaborators at each centre will independently code all timetabled global health learning events (compulsory, optional, or pre-elective), conforming to a pre-devised standard framework that will enable description of teaching events in terms of course year, duration, teaching format, and teaching content. The quantitative and qualitative data will be analysed using descriptive statistics and thematic analysis, respectively. Krippendorff's alpha coefficient will also be utilised to measure the level of agreement between datasets collected independently by different collaborators at the same centre.

\section{Results}

This study will define, for the first time, the nature and characteristics of global health education within UK medical school curricula. It will capture any differences in the amount of teaching provided on various global health learning outcomes between medical schools.

\section{Conclusions}

If this study depicts national discrepancies in teaching, it will place the onus on medical schools and the GMC to provide standardised and adequate global health education within the undergraduate and graduate entry medical curriculum. To aid medical schools in achieving this, the study will also identify currently scheduled teaching events at each medical school that could incorporate material related to the mandatory global health learning outcomes.

Global health can be best understood as the study, research, and practice of medicine focused on improving health and achieving health equity for all persons worldwide. $^{1-3}$ Such focuses are core tenets of modern medical practice. ${ }^{4,5}$ As health advocates, healthcare professionals
(HCPs) have an ethical responsibility to understand and engage with global issues, in order to improve health worldwide. ${ }^{6}$ It is key that this professional responsibility is fostered and encouraged at an early stage within medical education. ${ }^{7}$ Moreover, in today's interconnected world, 
health challenges transcend national boundaries. Globally, HCPs will be facing similar concerns, and collaborating to achieve resolution. A barrier to this transnational learning has been the lack of education on how health systems function across borders. ${ }^{8}$ An increased understanding of how care is delivered by various nations, is necessary for HCPs to learn from one another, and improve the practice of medicine globally. Furthermore, increased international migration and trade has accompanied increased globalisation. Consequently, diseases have been able to cross territorial boundaries more rapidly than ever before. ${ }^{9}$ For that reason, it is key for health professionals to understand international disease epidemiology and trends, in order to prevent the spread of epidemics. Equally, effective healthcare leadership involves focusing on whole population health. This necessitates an understanding of why ill health and health inequalities arise and persist in certain communities. ${ }^{10}$ To gain a greater awareness of patient and clinical complexities, it is important for HCPs to understand patients within the global context that they are embedded. Overall, it has become clear that global health education and training is essential to the core doctrines of modern-day medical professionals. 6

Over the course of the last century, the need for robust global health education within the UK medical training programme has received attention from media, students, ${ }^{11-13}$ and faculty alike.6,14-17 There are now legislative voices, both locally and internationally, who demand the restructuring of academic programmes - such as medicine - to include global health. ${ }^{18,19}$ There is a primary consensus that medical schools offer global health teaching in the form of optional special study modules or intercalated Bachelor of Science degrees. ${ }^{10,17}$ However, global health education has been mandated as an essential, non-elective component of medical school curricula. ${ }^{14,20,21}$ As the chief medical governing body, several documents from the General Medical Council (GMC): 'Tomorrow's Doctors', 'Outcomes for graduates 2018', and 'Generic professional capabilities framework $20,22,23$ outline specific global health learning outcomes and competencies to include and promote. ${ }^{14}$ Most recently, the Global Health Learning Outcomes Working Group applied the outcomes listed in Tomorrow's Doctors as a blueprint for key discussions with global health stakeholders, namely: universities, the public, Royal Colleges, and other professional, educational, and civil society bodies. Through this process, a comprehensive list of global health learning outcomes was derived for compulsory teaching at all UK medical schools. ${ }^{14}$ This list can be found in Appendix S1 of the Online Supplementary Document.

Despite strong arguments in favour of incorporating more global health teaching in the formal curriculum, there is a paucity of evidence demonstrating that medical schools have integrated these mandatory global health learning outcomes within their curricula. This is compounded by the limited data available from UK medical schools regarding the content of their medical syllabi, the methods through which they deliver educational material, and the quantity of global health education.

The Global Health Education in UK Medical Schools (GHEMS) study aims to delineate the breadth of global medicine teaching during undergraduate and graduate entry medical training across UK medical schools. This audit will evaluate current practices within UK medical curricula as compared to the Global Health Learning Outcomes Working group's comprehensive outcomes guide. Whilst the focus of this national audit will be on recording compulsory timetabled teaching, optional global health teaching and elective-related training will be recorded too. National discrepancies in teaching will be depicted, raising the onus on medical schools to provide a standardised and adequate medical education in global health. This model may also guide related ventures and undertakings on how teaching differences relate to university infrastructure, entry requirements, student perceptions, outcomes in Foundation and speciality application examinations, and career paths.

\section{METHODS}

\section{PRIMARY AIM}

To evaluate the extent of timetabled global health teaching activities in UK medical schools.

\section{SECONDARY AIMS}

- To characterise the nature and characteristics of global health education within medical school curricula.

- To develop a valid and reliable model that can be used henceforth to audit and evaluate medical education.

- To identify scope for expanded integration of compulsory global health education within medical school curricula.

- To provide advocacy for the need for global health education in UK medical school curricula.

- To establish a collaboration of medical students interested in global health to help enhance research capacity and to create a platform for future collaborative work.

\section{STUDY DESIGN}

GHEMS is a national, multi-centre audit evaluating global health education within UK medical school curricula during the academic year 2018/19. Global health education at UK medical schools will be compared to guidelines - derived from the GMC's recommendations - published by the Global Health Learning Outcomes Working Group for compulsory teaching at all UK medical schools. ${ }^{14}$ The study will be disseminated through collaborative university medical school and student networks. The generic collaborative method, and the benefits that participating students derive from it are based on previous well-documented literature. ${ }^{21,24-26}$

The GHEMS study will be delivered by the UK division of the International Student Surgical Network (InciSioN). InciSioN UK is a student-led, national body that aims to empower medical students and junior doctors in the UK to undertake global health research, education and advocacy. 


\section{STUDY SETTING}

All medical schools in the United Kingdom recognised by the GMC, where students are currently studying for a UK medical degree, will be eligible to participate. A list of these medical schools can be found in Appendix S2. Medical schools that are scheduled to start after the period of this study (Universities of Sunderland, Lincoln and Edgehill) have been excluded. Medical schools located in the UK, with medical qualifications awarded by an overseas university, have also been omitted.

Each centre will provide data for all timetabled global health teaching activities carried out within the academic year $2018 / 2019$, for each year of medical education in their undergraduate and/or graduate entry medical courses. This will ensure a full representation of undergraduate and graduate entry global health education in the UK.

\section{COLLABORATOR INFORMATION}

Any medical student enrolled in one of the medical schools in Appendix S2 of the Online Supplementary Document is eligible to participate as a collaborator in the study. Medical students will be invited to become collaborators in the study through several routes:

- Medical Societies.

- Medical School mailing lists.

- Organisations focused on global health, including Lifebox, InciSioN, and Students for Global Health.

- Conferences.

- Social media.

The maximum number of collaborators from each medical school is two. If more than two applications are received from a medical school, the GHEMS steering committee will score the applications based on their merit, and the top two ranking applicants will be selected.

Once selected, collaborators must attend a centralised training and support session delivered by the GHEMS steering committee. Following the training session, collaborators will be responsible for ensuring the correct departmental approval has been achieved prior to commencing data collection. Evidence of approval must be sent to the GHEMS steering committee prior to commencement of data collection. It is key that medical schools are formally engaged at an early stage of this study.

Following approval, collaborators must obtain timetables containing details of all medical teaching provided at undergraduate and/or graduate entry level at their medical school for the academic year 2018/2019. Each collaborator will independently code all timetabled global health learning events (compulsory, optional, or pre-elective), conforming to a pre-devised standard framework that will enable description of teaching events in terms of course year, duration, teaching format, and teaching content. The suggested overall data collection period will be Monday $1^{\text {st }}$ April 2019 to Monday $1^{\text {st }}$ June 2019 . Each collaborator must collect the data independently. Following data collection, the data will be validated by the GHEMS steering committee, who will evaluate the level of agreement between datasets collected independently by different collaborators at the same centre. Table 1 shows the timeline for this project.

Following completion of the GHEMS study, all collaborators will be encouraged to present their local findings to the faculty members responsible for organising the curriculum at their medical school. This is an essential step in all audit loops. In addition, presenting local results will help collaborators develop analytical and presentation skills, review the results with their local institutions, and encourage reformation from the process. Medical schools will also be able to request their own specific data and the analysis done on said data from the GHEMS steering committee following study completion.

\section{DATA COLLECTION}

If two collaborators are present in one medical school, they may act as a team for steps 1 and 2. For steps 3-19, they must collect the data independently and must not confer with one another.

1. Gain approval for data collection from your local institution. It is recommended that this be done as a first step, by reaching out to your head of curriculum, through email. A template email - Appendix S3 in the Online Supplementary Document- that includes all the relevant information will be circulated to local collaborators.

2. Obtain complete timetables for all undergraduate and graduate entry medical teaching for the academic year 2018/ 2019 at your medical school. For intercalated years, please obtain the timetable for the subject offered by your university that offers the most global health teaching.

3. Establish the learning objectives for each timetabled event. For a timetabled student selected component that offers a range of options, please obtain the learning objectives for the option that offers the most global health teaching. If learning objectives are not available for a timetabled event, skip step 4 . If the timetabled event is an elective, go straight to step 19.

4. Determine which learning objectives indicate an explicit global health element. Select these learning objectives. From the learning objectives that have not yet been selected, determine which ones correspond with a broad non-global health specific version of the outcomes noted in Appendix S1. Select these learning outcomes also. For example, a learning objective focused on maternal health would be determined not to indicate an explicit global health element. However, it should be deemed to correspond with the broad version of the 'maternal, reproductive, and child health of various countries' outcome, as the 'various countries' aspect should not be considered for this step. Therefore, the learning objective would be selected.

5. Identify and select timetabled teaching events that do not have learning objectives available, or whose learning objectives were selected in step 4 .

6. Populate the data collection form - detailed in Appendix S4 - with the name of all teaching events selected in step 5 . 
Table 1. Project timeline*

\begin{tabular}{ll}
\hline Dates & Description \\
\hline $\begin{array}{l}1^{\text {st }} \text { December } 2018-31^{\text {st }} \\
\text { December } 2018\end{array}$ & Pilot study is done \\
$1^{\text {st }}$ January 2019 & GHEMS collaborator recruitment opens \\
$1^{\text {st }}$ February 2019 & General GHEMS collaborator recruitment closes \\
$1^{\text {st }}$ March 2019 & GHEMS collaborator training and support sessions commence \\
$1^{\text {st }}$ April 2019 & Start of data collection period \\
$1^{\text {st } J u n e ~} 2019$ & Final data submission deadline \\
$2^{\text {nd }}$ June $2019-14^{\text {th }}$ July 2019 & Data validation and data analysis will take place \\
$15^{\text {th }}$ July 2019 onwards & GHEMS data should be presented to faculty members responsible for organising the \\
\hline
\end{tabular}

*Project timeline - extended data collection periods may be incorporated to grant flexibility to centres that may have experienced logistical obstacles to study commencement.

7. Populate the data collection form with the year of medical training at which the teaching events selected in step 5 occur. If a medical school has a mandatory intercalated year, the years on either side should be consecutively numbered for the purposes of this data collection eg, year 2 , intercalated year, year 3 , etc.

8. Populate the data collection form with the amount of time it took to conduct each teaching event selected in step 5.

9. Populate the data collection form with the teaching format (eg, compulsory teaching, student selected component, or pre-elective training) of all teaching events selected in step 5 .

10. Populate the data collection form with the style of teaching (eg, PBL, lecture, small group, self-directed) for all teaching events selected in step 5 .

11. Obtain the content for each teaching event selected in step 5. If unable to access the content of a teaching event, please skip steps 12-15.

12. Go through the content obtained in step 11 and identify all material that have a global health element to them.

13. Use the information collected in steps 4 and 12 to fill the data collection form with the description of the teaching content for all teaching events selected in step 5 . This involves writing down the learning objectives selected in step 4, and the material identified in step 12.

14. In the data collection form, write yes in the box related to an outcome noted in Appendix S1 of the Online Supplementary Document if the teaching event has met the criteria of that point.

15. If the teaching event has not met a outcome noted in Appendix S1 of the Online Supplementary Document, leave the box related to that point blank in the data collection form.

16. If you are unable to access the content of the teaching event selected in step 5, please write 'inaccessible' when filling the description of the teaching content in the data collection form. In the same box, please also write down the learning objectives of said teaching event that were selected in step 4 .
17. If step 16 was used for a teaching event, use secondhand accounts of said teaching event where possible to note down whether any global health content was covered. Place this information in the 'description of the teaching content' section of the data collection form, and preface it with 'secondary sources claim:'

18. If pre-elective training was selected for a teaching event in step 9, please also note down whether any materials or services are provided to students to assist them with elective planning.

19. If the timetabled event is an elective, write down 'elective' as the name of the teaching event, write down which year of medical training the elective took place in, and write down the number of weeks a medical student can go on elective for in the data collection form. If debriefing routinely occurs following an elective at your medical school, please describe the material covered in the 'description of the teaching content' section of the data collection form.

\section{PILOT STUDY}

A pilot study was carried out by medical students at the Universities of Oxford, Warwick and Sheffield. This mini study was done in order to optimise the design of the data collection form, and ensure methodology was feasible, with no barriers to effective data collection or study completion. A flowchart of the methods employed by the collaborator at each university is presented in Figure 1. A consensus discussion agreed that the method used for Warwick Medical School was the best template for collaborators to use for this study.

\section{PRIMARY OUTCOME}

The primary outcome is compliance with the gold standard guidelines - derived from the GMC's recommendations published by the Global Health Learning Outcomes Working Group for compulsory global health teaching at all UK medical schools. ${ }^{14}$ The primary outcome measure will be the proportion of the 42 global health learning outcomes listed in Appendix S1 - taught as part of compulsory timetabled teaching in the undergraduate and graduate entry medical training across UK medical schools. Proportions 
A

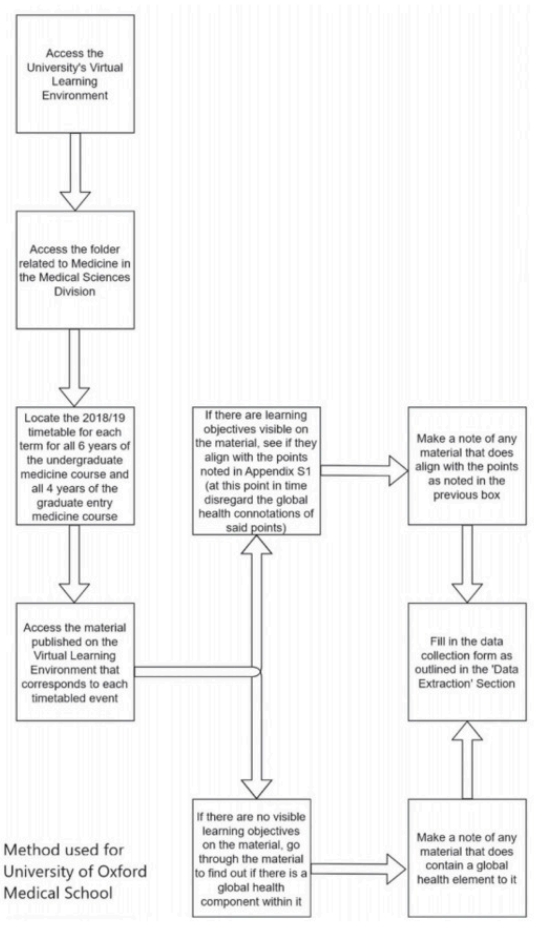

B

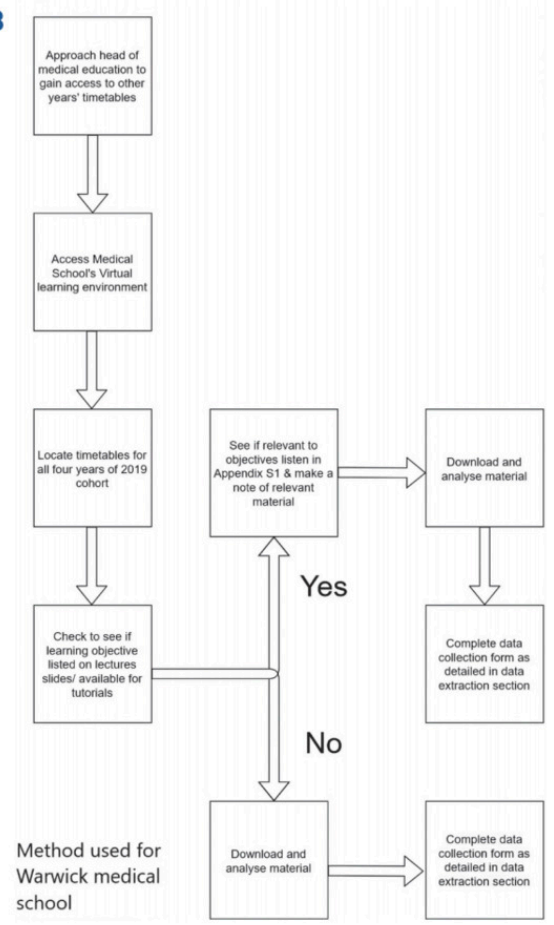

C

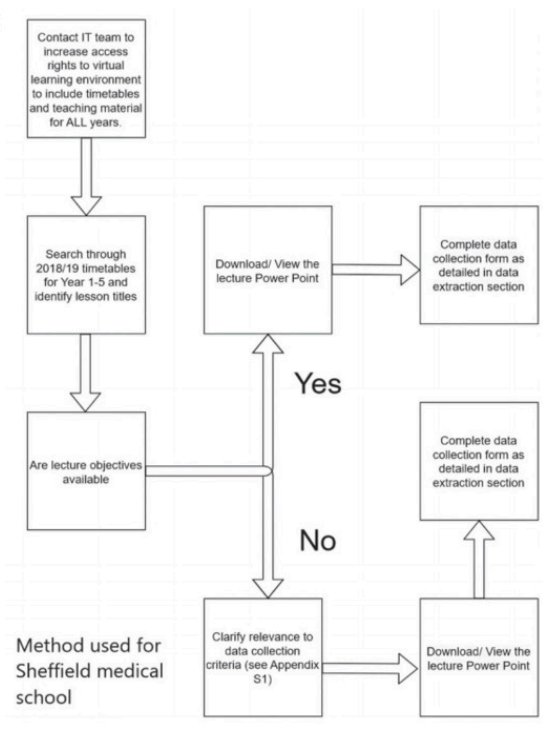

Figure 1. Flowchart of pilot studies conducted at a) Oxford, b) Warwick, and c) Sheffield medical schools. 
(P) will be calculated by counting the number of global health learning outcomes met by at least one compulsory timetabled teaching station - each global health learning outcome will only be counted once - and dividing that number by 42 . The results will be anonymised, and no centre-specific comparisons will be performed. Subgroup analyses may be performed to look at differences between global health education delivered at compulsory 6-year courses, 5-year courses, and 4-year graduate entry courses.

\section{SECONDARY OUTCOME}

Secondary outcome measures will include:

- Proportion of the 5 global health themes covered by compulsory timetabled teaching in the undergraduate and the graduate entry medical courses of UK medical schools.

- Proportion of the 17 global health sub-themes taught as part of compulsory timetabled teaching in the undergraduate and the graduate entry medical courses of UK medical schools.

- Proportion of timetabled events with learning objectives similar to outcomes in Appendix S1 that do not currently possess global health material at each UK medical school.

- The number of weeks of elective availability for students at each UK medical school.

- The number of hours of global health teaching that all students are guaranteed to receive at each UK medical school.

- To describe the structure of global health education at each UK medical school.

\section{QUALITY ASSURANCE}

Design: This protocol and the accompanying appendices were designed and compiled with guidance from an expert cross-specialty advisory group. The methodology for student-driven audits and service evaluation have been widely validated across multiple datasets nationally and internationally demonstrating high levels of data accuracy. ${ }^{27-31}$

Training: All participating collaborators will attend a mandatory centralised training and support session delivered by the GHEMS steering committee. This session will allow investigators to become familiar with the protocol and describe how to register and run the study at their medical school. It will also allow collaborators to provide feedback on local issues or questions raised by their medical school. Throughout the data collection period, the GHEMS steering committee will be in regular contact will all the collaborators. This should allow collaborators to get prompt clarifications on any uncertainties that arise during the course of the project.

Data completeness: Following data collection, only datasets with $>95 \%$ of data completeness will be accepted. To emphasise the importance of data completeness to collaborators, data collections with $>5 \%$ missing data points will be excluded from the study and collaborators withdrawn from the published list of citable collaborators.

Validation: Medical students at the Universities of Oxford, Warwick, and Sheffield carried out a pilot study. Based on their experiences, the data collection form was modified to improve clarity, objectivity, and more accurately measure the extent of timetabled global health teaching activities in UK medical schools. The data collection form was then sent to medical students at the Universities of Edinburgh, Leicester, King's College London, Birmingham, and Leeds. These medical students were selected as they had not been involved in creating the data collection form. Their responses were evaluated for potential problems, and the form was updated to ensure there were no barriers to effective data collection and study completion across participating centres. The protocol was also updated to include processes used in the pilot study to access teaching content. Validating steps were also built into the data collection protocol and data collection form. For example, timetabled events without learning objectives are automatically included for further analysis to avoid missing any material that addresses an outcome listed in Appendix S1 of the Online Supplementary Document. Similarly, if the content of a teaching event is inaccessible, 'inaccessible' will be entered when filling the description of the teaching content in the data collection form. However, we have included processes in the protocol with the aim of gaining an insight into the content of these teaching sessions.

In addition, two collaborators will be recruited to collect data at most medical schools participating in the GHEMS study. During the period of data collection, collaborators collecting data from the same centre will independently code all timetabled global health learning events and will not confer with one another. Collaborators will be reminded of this requirement through regular communications. Following data collection, Krippendorff's alpha coefficient will be utilised to measure the level of agreement between datasets collected independently by different collaborators at the same centre: the inter-rater reliability. Similarly, the medical students at the Universities of Oxford, Warwick, and Sheffield who carried out the pilot study will be asked to repeat their data collection. Following data collection, independent assessors - medical students not involved in the initial data collection and university faculty members - will determine the degree to which the two data sets collected at different times agree: intra-rater reliability. Furthermore, all collaborators will be asked to create a report regarding their experience with collecting data in order to identify any potential areas for error and to aid with data interpretation.

\section{DATA MANAGEMENT}

A standardised Microsoft Excel spreadsheet (Excel 2010; Microsoft, Redmond, Washington, USA) with pre-set fields will be used to collect data at each centre. Data protection regulations at each centre will be complied with. The audit does not involve the collection of any personal data. All data collected will be anonymised post-hoc. The data governance was approved following a review by a research ethicist and the director of medical ethics at Oxford Medical School. 


\section{STATISTICAL ANALYSIS}

The resultant data will be analysed via descriptive statistics to determine the proportion of the 42 global health learning outcomes published by the Global Health Learning Outcomes Working Group that have been integrated into undergraduate and graduate entry medical training at each UK medical school. IBM SPSS Statistics version 24.0 software will be employed for data analysis and to obtain descriptive summary statistics and outcomes. With the assistance of a qualitative researcher, we will aim to utilise a deductive thematic analysis approach to qualitatively analyse the data contained within the 'description of teaching content' section of the data collection form.

\section{AUTHORSHIP}

In accordance with National Research Collaborative (NRC) authorship guidelines, ${ }^{24}$ all publication outputs from GHEMS will be listed under a unified corporate authorship: 'InciSioN UK Collaborative'. Certain publications will include named authors on the bye-line as well as the group name. This will follow the example set by commendable collaborative including STARSurg and GlobalSurg. ${ }^{25-27,29-31}$ Anyone who has demonstrated satisfactory completion of the minimum requirements for authorship will be eligible for PubMed-citable collaborative authorship in accordance with the roles defined below:

Writing Group: Responsible for the overall scientific content, data analysis, and preparation of research manuscripts.

Steering Committee: Responsible for the protocol design, project coordination, and data handling.

External Advisory Group: Cross-disciplinary experts who can ensure contextual and scientific relevance of the protocol design, data fields, and data interpretation.

Collaborators: A network of medical students across all medical schools. They are responsible for data collection.

Ethics and dissemination

\section{ETHICS}

According to King's College London Research Ethics Committee guidelines (Appendix S5), the study is classified as an audit, and hence does not require ethical approval. This has been confirmed following a review by a research ethicist and the director of undergraduate medical ethics at the University of Oxford.

The study fulfils the audit criteria and does not need ethical approval due to the following reasons:

- All data collected will measure current compulsory global health teaching delivered in UK medical schools.

- Current teaching standards will be compared to published standards in the literature ${ }^{14,20,20,23}$

- The study does not involve interventions or randomisations to the delivery of medical education.

- No individual collaborator or medical school taking part will be independently identifiable in the study results
- Personal information will not be collected in this study

- No data will be recorded by a collaborator prior to written (or electronic/email) approval from the medical school they are collecting data from

- Data protection regulations at each centre will be complied with

Additionally, advice was sought from King's College London Research Ethics Committee and Oxford Medical Sciences Inter-Divisional Research Ethics Committee. Confirmation has been been provided that the study does not require ethical approval (Appendices S6 and S7). Local approval must be sought accordingly.

\section{DISSEMINATION}

The protocol will be disseminated primarily through recruited medical student collaborators. Should UK medical schools wish to see the protocol, collaborators may pass it along as well. The study protocol will also be submitted for peer-reviewed publication. Any publications of the protocol will be advertised through social media, including Twitter and Facebook.

Following study completion, teleconferences will be held with all collaborators to share and discuss the data analysis undertaken and the study results. Following this, the results will be presented at local, regional, national, and international conferences by medical student collaborators. A standard PowerPoint presentation and poster will be created for this purpose. All presentations will be coordinated by the GHEMS steering committee to adhere to conference guidelines and to ensure all conference regulations are fulfilled. In addition, the results will be disseminated via publication in a peer-reviewed medical journal. All collaborators will be co-authors of resulting presentations and PubMed Citable co-authors of resulting publication(s).

Following publication, the manuscript can be shared by collaborators with their medical schools to feedback the study results, and to highlight the scope for expanded integration of compulsory global health education into the existing curricula. Medical schools may request their own specific data and the analysis done on said data from the GHEMS steering committee following study completion.

\section{DISCUSSION}

The study has been designed to be delivered through a national student collaborative group, with the aim of engaging students with multicentre studies and global health and establishing an InciSioN UK collaborative for future collaborative work. The methodology for collaborative student-led groups has been widely validated across multiple datasets nationally and internationally and has a track record for producing high-quality data. ${ }^{27-31}$ A comprehensive study protocol and centralised training will ensure consistent and reproducible data collection. Additionally, fulfilment of collaborator roles will grant individuals with collaborator authorship, in accordance with NRC authorship guidelines, ${ }^{24}$ and may stand as an incentive to achieve high data completion rates. Recruiting two collaborators per medical school 
to independently assess the same curriculum will also increase the reliability of our outcome measures.

The study will be geographically comprehensive, as all 37 medical schools that are recognised by the GMC, where students currently study for a UK medical degree, will be eligible to participate (Appendix S2 of the Online Supplementary Document). We anticipate that recruitment will engage students from at least $80 \%$ of these medical schools, and there will be two collaborators present in at least $80 \%$ of participating medical schools. Medical schools that are scheduled to start after the period of this study (Universities of Sunderland, Lincoln and Edgehill) and medical schools located in the UK, with medical qualifications awarded by an overseas university, have been excluded from the study.

The limitations of this study relate to its snapshot observational design, which restricts the volume and complexity of data that can be collected. For example, we are unable to assess how global health teaching has changed from academic year to year. Our data collection is representative of the 2018/2019 curriculum, and we acknowledge that within the span of medical school for an individual, that curriculum changes are eminent. Additionally, to procure the data, collaborators may rely on learning objectives and teaching content available in a university's virtual learning environment. However, the content and learning objectives uploaded may differ from the true content delivered at a timetabled teaching event. Similarly, there may be learning objectives available for non-timetabled teaching events - such as teaching that happens on the wards - that correspond with a global health learning outcome published by the Global Health Learning Outcomes Working Group. Global health education also involves time spent in health care settings in different parts of the world through elective attachments, summer projects, global health conferences and other such platforms. We acknowledge that such opportunities vary between medical schools, and they have not been assessed thoroughly by this study. Similarly, this study does not assess whether the global health learning outcomes are examined at medical schools, and therefore whether competency in them are achieved by medical students.

This multicentre study will define, for the first time, the nature and characteristics of global health education within UK medical school curricula. If this study depicts national discrepancies in teaching, it will place the onus on medical schools and the GMC to provide standardised and adequate global health education within the undergraduate and graduate entry medical curriculum. To aid medical schools in achieving this, the study will also identify currently scheduled teaching events at each medical school that could incorporate global health learning outcomes published by the Global Health Learning Outcomes Working Group. ${ }^{14}$ In addition, we will highlight discrepancies in the amount of teaching provided on each of the 42 global health learning outcomes between medical schools, by recording the du- ration of teaching and the global health content delivered during each timetabled session. The GHEMS model may also guide related ventures and undertakings on how medical education relates to university infrastructure, entry requirements, student perceptions, outcomes in Foundation and specialty application examinations, and career paths.

\section{ACKNOWLEDGEMENTS}

Roba Khundkar, Riana Patel, David Clark, Michael Dunn, Oliver Johnson, Ewen Harrison, Bridget Young, Nusrat Husain, Dmitri Nepogodiev, Parivrudh Sharma, Pat Lok, Catherine Dominic, Abigail Jamieson, Felicity Greenfield, Areeba Kabir, Anmol Arora, Ahmed Bilal, Maarja-Liis Ferry, James McLaren, Saud Alfadhel, Bonita Sinartio, Nitish Nachiappan, Hassan Muha, Mariam Gaddah, Biyyam Meghna Rao, Won Young Yoon, Katarina Hoernke, Tom Poundall, Luisa Hofmaier, James Bevan. Kiana Bowden, Farhiya Omar, Belle Liew, Shahnoor Minhas Amin, Amir Mohammed, Sally Hashim Yousif Bayoumi Mohammed, Lotta Gustafsson, Eleanor Deane, Flora Jobson, Rhys Wenlock, Jack Wellington, Chung Sien Chai, Emma Sharland, Beatrice Prosser, Agata Oliwa, Amy Gomes, Connor Moore, Latifa Haque, Jordan Delong, Alan Penney, Asveny Rajananthanan, Anuradha Ponnapalli, Isabella Burns, Annabel Killen, Arina Toma, Heather Lawson, Morgan Weiland, Chiara Cotronei, Mariam Ahmed, Emel Yildirim, Ankit Bhatt, Dilan Parmar on behalf of the InciSioN UK collaborative group.

\section{FUNDING}

No funding has been provided for this study.

\section{COMPETING INTERESTS}

JEF is Honorary Clinical Advisor for the LifeBox Foundation (unpaid), Trustee of the Surgical Research Gateway (SURG) Foundation (unpaid), and a consultant for the global healthcare practice at KPMG International (paid). All remaining authors completed the Unified Competing Interest form at http://www.icmje.org/coi disclosure.pdf (available upon request from the corresponding author), and declare no further conflicts of interest.

\section{CORRESPONDENCE TO:}

Mr Soham Bandyopadhyay BA

St Hilda's College

Cowley Place

Oxford, OX4 1DY

UK

Soham.bandyopadhyay@yahoo.co.uk 


\section{REFERENCES}

1. Koplan JP, Bond TC, Merson MH, et al. Towards a common definition of global health. Lancet. 2009;373(9679):1993-1995. doi:10.1016/s0140-6736(0 9)60332-9

2. Kickbusch I. The need for a European Strategy on Global Health. Scand J Public Health. 2006;34(6):561-565. doi:10.1080/14034940600973059

3. Beaglehole R, Bonita R. What is global health? Glob Health Action. 2010;3(1):5142. doi:10.3402/gha.v3i0.5 $\underline{142}$

4. NHS Employers. The Role of the Doctor Consensus statement. Published 2009. Accessed March 15, 2019. https://www.nhsemployers.org/-/media/Employers/D ocuments/Plan/consensus_statment_medical_workfor ce.pdf

5. General Medical Council. The duties of a doctor registered with the General Medical Council. Published 2001. Accessed March 15, 2019. https://ww w.gmc-uk.org/ethical-guidance/ethical-guidance-fordoctors/good-medical-practice/duties-of-a-doctor

6. Frenk J, Chen L, Bhutta ZA, et al. Health professionals for a new century: transforming education to strengthen health systems in an interdependent world. Lancet.

2010;376(9756):1923-1958. doi:10.1016/s0140-6736(1 0)61854-5

7. Hilton SR, Slotnick HB. Proto-professionalism: how professionalisation occurs across the continuum of medical education. Med Educ. 2005;39(1):58-65. doi:1 0.1111/i.1365-2929.2004.02033.x

8. Quam L, Smith R. What can the UK and US health systems learn from each other? BMJ. 2005;330(7490):530-533. doi:10.1136/bmj.330.7490.5 $\underline{30}$

9. World Health Organization. Globalization and Infectious diseases: A review of the linkages. Published 2004. Accessed March 9, 2019. https://ww w.who.int/tdr/publications/documents/seb topic3.pdf

10. Watson J, Shiner A, Pettigrew LM, Irving G. Global health: time for full integration into GP education. $\mathrm{Br}$ J Gen Pract. 2013;63(610):271-272. doi:10.3399/bigp1 $\underline{3 \times 667358}$

11. Hilgers J, De Roos P, Rigby E, Hilgers J, De Roos P, Rigby E. European Core Curriculum-the Students' Perspective, Bristol, UK, 10 July 2006. Med Teach. 2007;29(2-3):270-275. doi:10.1080/014215907012687 31
12. Stigler FL, Duvivier RJ, Weggemans M, Salzer HJ. Health professionals for the 21st century: a students' view. Lancet. 2010;376(9756):1877-1878. doi:10.1016/ s0140-6736(10)61968-x

13. Khan OA, Guerrant R, Sanders J, et al. Global health education in U.S. Medical schools. BMC Med Educ. 2013;13(1):3. doi:10.1186/1472-6920-13-3

14. Johnson O, Bailey SL, Willott C, et al. Global health learning outcomes for medical students in the UK. Lancet. 2012;379(9831):2033-2035. doi:10.1016/s 0140-6736(11)61582-1

15. Hall J, Brown CS, Pettigrew L, et al. Global health in UK postgraduate medical training. Lancet. 2012;380(9843):728-729. doi:10.1016/s0140-6736(1 2)61404-4

16. Irving G, Riley B, Rughani A, et al. Global health in the UK's GP postgraduate curriculum. Lancet. 2012;380(9854):1646-1647. doi:10.1016/s0140-6736(1 2) $61947-3$

17. Martineau F, Johnson O, Rowson M, Willott C, Yudkin JS. International health graduates-career path experience. Lancet. 2012;379(9831):2051-2052. d oi:10.1016/s0140-6736(12)60893-9

18. Institute of Medicine. The U.S. Commitment to Global Health: Recommendations for the U.S. Government. National Academies Press (US); 2009.

19. UK Parliament. Immigration: High Court Ruling. Published 2010. Accessed March 1, 2019. https://publi cations.parliament.uk/pa/ld201011/ldhansrd/text/101 220-0002.htm

20. General Medical Council. Tomorrow’s Doctors. Published 2009. Accessed March 1, 2019. http://ww w.ub.edu/medicina_unitateducaciomedica/document os/TomorrowsDoctors_2009.pdf

21. Brewer TF, Saba N, Clair V. From boutique to basic: a call for standardised medical education in global health. Med Educ. 2009;43(10):930-933. doi:1 $\underline{0.1111 / j .1365-2923.2009 .03458 . x}$

22. General Medical Council. Generic professional capabilities framework. Published 2017. Accessed March 1, 2019. https://www.gmc-uk.org/-/media/doc uments/generic-professional-capabilities-framewor k--0817_pdf-70417127.pdf 
23. General Medical Council. Outcomes for graduates 2018. Published 2018. Accessed March 1, 2019. http s://www.gmc-uk.org/-/media/documents/dc11326-ou tcomes-for-graduates-2018_pdf-75040796.pdf

24. National Research Collaborative \& Association of Surgeons in Training Collaborative Consensus Group. Recognising contributions to work in research collaboratives: Guidelines for standardising reporting of authorship in collaborative research. Int J Surg. 2018;52:355-360. doi:10.1016/j.ijsu.2017.12.01929292 $\underline{217}$

25. Bhangu A, Fitzgerald JE, Kolias AG. Trainee-led research collaboratives: a novel model for delivering multi-centre studies. ANZ J Surg.

2014;84(12):902-903. doi:10.1111/ans.12797

26. Chapman SJ, Glasbey JCD, Khatri C, et al. Promoting research and audit at medical school: evaluating the educational impact of participation in a student-led national collaborative study. BMC Med Educ. 2015;15(1):47. doi:10.1186/s12909-015-0326-1

27. STARSurg Collaborative. Prognostic model to predict postoperative acute kidney injury in patients undergoing major gastrointestinal surgery based on a national prospective observational cohort study. BJS Open. 2018;2:400-410. doi:10.1002/bjs5.8630513129
28. STARSurg Collaborative. Impact of postoperative non-steroidal anti-inflammatory drugs on adverse events after gastrointestinal surgery. Br J Surg. 2014;101:1413-123. doi:10.1002/bjs.961425091299

29. STARSurg Collaborative. Multicentre prospective cohort study of body mass index and postoperative complications following gastrointestinal surgery. $\mathrm{Br} \mathrm{J}$ Surg. 2016;103:1157-1172. doi:10.1002/bjs.102032732 $\underline{1766}$

30. EuroSurg Collaborative. Body mass index and complications following major gastrointestinal surgery: a prospective, international cohort study and meta-analysis. Colorectal Dis. 2018;20:0215-25. doi:1 $\underline{0.1111 / \text { codi.1429229897171 }}$

31. GlobalSurg Collaborative. Surgical site infection after gastrointestinal surgery in high-income, middle-income, and low-income countries: a prospective, international, multicentre cohort study. Lancet Infect Dis. 2018;18:516-525. doi:10.1016/S147 3-3099(18)30101-429452941 


\section{SUPPLEMENTARY MATERIALS}

\section{Online Supplementary Document}

Download: https://www.joghr.org/article/12011-global-health-education-in-uk-medical-schools-ghems-studyprotocol/attachment/36738.pdf 